\title{
Dynamic Behavior of Cluster Observables for the 2d Ising Model
}

\author{
Wanderson G. Wanzeller ${ }^{a}$, Tereza Mendes ${ }^{b}$, and Gastão Krein ${ }^{a}$ \\ ${ }^{a}$ Instituto de Física Teórica, Universidade Estadual Paulista \\ Rua Pamplona 145, 01405-900, São Paulo, SP, Brazil \\ ${ }^{b}$ Instituto de Física de São Carlos - Universidade de São Paulo \\ C. P. 369, 13560-970, São Carlos, SP, Brazil
}

Received on 01 October, 2005

\begin{abstract}
We present results of our numerical study of the critical dynamics of percolation observables for the twodimensional Ising model. We consider the (Monte Carlo) short-time evolution of the system with small initial magnetization and heat-bath dynamics. We find qualitatively different dynamic behaviors for the magnetization $M$ and for $\Omega$, the so-called strength of the percolating cluster, which is the order parameter of the percolation transition. More precisely, we obtain a (leading) exponential form for $\Omega$ as a function of the Monte Carlo time $t$, to be compared with the power-law increase encountered for $M$ at short times. Our results suggest that, although the descriptions in terms of magnetic or percolation order parameters may be equivalent in the equilibrium regime, greater care must be taken to interpret percolation observables at short times.
\end{abstract}

Keywords: Critical short-time dynamics; Percolation; Ising model

\section{INTRODUCTION}

For many physical systems, a suitable definition of cluster provides a mapping of the physical phase transition into the geometric problem of percolation, allowing a better understanding of how the transition is induced in the system. For Ising and $n$-vector spin models this mapping is well understood [1], whereas for lattice gauge theories it may be harder to define, even in the simpler pure-gauge case [2]. In the case of the Ising model, the physical (droplet) clusters coincide with the ones used for global spin updates in the Swendsen-Wang Monte Carlo algorithm (i.e. the ones introduced by Coniglio and Klein, based on the prescription by Fortuin and Kasteleyn [3]). These are the clusters we consider here, although our Monte Carlo updates are given by a local heat-bath algorithm.

Given a definition for a cluster on the lattice, the order parameter in percolation theory is the so-called strength of the percolating cluster $\Omega$, defined by

$$
\Omega \equiv\left\{\begin{array}{cc}
0, & T>T_{c} \\
\Delta / V, & T \leq T_{c}
\end{array}\right.
$$

where $\Delta$ is the volume of the percolating cluster and $T_{c}$ is the critical temperature. (Note that a percolating cluster is a set of spins connected from the first to the last row of the lattice.)

When equilibrium properties are investigated, the descriptions in terms of the magnetic order parameter $M$ or the percolation order parameter $\Omega$ are indeed equivalent (see, for example, $[4,5]$ and references therein) and one finds the same critical exponents. Thus one might expect to find equivalence for dynamic quantities as well, and in particular for the behavior at short times.

In the present work[11] we investigate the dynamic critical behavior for the two-dimensional (zero-field) Ising model

$$
\mathcal{H}=-J \sum_{<i, j>} S_{i} S_{j}
$$

at short Monte Carlo time $t$, using the (local) heat-bath algorithm. The observables measured are the magnetization $M=1 / V \sum_{i} S_{i}$ and the percolation order parameter $\Omega$ defined above. We compare the behaviors of the two quantities as functions of $t$ and find that, whereas the magnetic order parameter shows a power-law increase with $t$, the data for the percolation order parameter $\Omega$ are well fitted by a sum of exponentials of the (inverse of the) time $t$. At the end of Section III we also comment on the short-time behavior of another definition for the percolation order parameter, based on the largest cluster instead of the spanning (percolating) cluster.

\section{SHORT-TIME (MONTE CARLO) DYNAMICS}

Using renormalization-group theory, it can be shown [7] that the early time evolution of an order parameter (e.g. the magnetization $M$ ) already displays universal critical behavior, given by

$$
M\left(t, \varepsilon, m_{0}\right)=b^{-\beta / v} \mathcal{M}\left(t b^{-z}, \varepsilon b^{1 / v}, m_{0} b^{x_{0}}\right),
$$

where $m_{0}$ is the initial magnetization, $\varepsilon \equiv\left(T-T_{c}\right) / T_{c}, \mathcal{M}$ is a universal function and $b$ is a scale factor, which can be taken equal to $t^{1 / z}$. We thus expect for $T=T_{c}$ and small $m_{0}$ a powerlaw behavior at early times $M(t)_{\mathcal{\varepsilon} \rightarrow 0} \sim m_{0} t^{\theta}$, with $\theta=\left(x_{0}-\right.$ $\beta / v) / z$. In principle, we would assume that the percolation order parameter $\Omega$ should have a similar behavior. The time evolution for the heat-bath algorithm and the evaluation of the Swendsen-Wang clusters are described below.

The heat-bath dynamics consists in choosing the two possible directions of each Ising spin according to the exact conditional probability given by its nearest neighbors. Each spin $S_{i}$ is chosen to point $u p$ with probability $p_{i}$, or down with probability $1-p_{i}$, where

$$
p_{i}=\frac{1}{1+\exp \left(-2 \beta J \sum_{j} S_{j}\right)} .
$$


Note that $\beta=1 / K T$ and the sum is over nearest neighbors of $S_{i}$. After a certain number of iterations the spin configuration obeys the Boltzmann distribution. In the heat-bath method, since the updates are local, this transient time becomes considerably large at criticality.

The Swendsen-Wang (cluster) algorithm is obtained from the Ising-model Hamiltonian by writing the partition function as

$$
Z=\sum_{\{S\}} \sum_{\{n\}}\left\{\prod_{\langle i, j\rangle}^{n_{i j}=1} p_{i j} \delta_{S_{i} S_{j}}\right\}\left\{\prod_{\langle i, j\rangle}^{n_{i j}=0}\left(1-p_{i j}\right)\right\}
$$

where $p_{i j}=1-\exp (-2 \beta J)$ is the probability of having a link between two nearest-neighbor sites of equal spin value. This link is represented by $n_{i j}$ and determines the clusters that will be associated with percolation at the critical temperature. Note that in the Swendsen-Wang algorithm one performs global moves in which the spins in a cluster are flipped together. Here we only use the clusters just defined to calculate percolation observables, whereas the dynamics is given by local heat-bath updates, as described above.

\section{NUMERICAL RESULTS}

In order to study the short-time dynamics we simulate at $T=T_{c}$ and force the system to have an initial magnetization $m_{0}$. We let the system evolve in time and look for power-law behavior of the order parameters $M$ and $\Omega$ as a function of the (Monte Carlo) time. Each temporal sequence is generated from a different random seed, i.e. each sequence has a different initial spin configuration. The time history is then obtained from an average over all the generated sequences. We have performed Monte Carlo simulations with 50000 seeds and 5000 sweeps for three initial magnetizations $\left(m_{0}=0.02\right.$, $0.03,0.04)$, for four lattice volumes, using the heat-bath algorithm.

In Fig. 1 we show the comparison between the early-time behaviors of $M$ and $\Omega$ for a $250 \times 250$ lattice, starting from an initial magnetization $m_{0}=0.02$.

We see that although the two curves approach the same equilibrium value, their early time increase is qualitatively different. We obtain that a power-law fit works very well for $M$, yielding the literature value [8] for the exponent $\theta$. Our results for $\theta$ are shown in Table I below.

The data for $\Omega$, on the other hand, are clearly not well described by a power law. We have found that a (3-parameter) fit to the form

$$
\Omega=a \exp (-\tau / t)+b \exp (-2 \tau / t)
$$

works well, yielding the results shown in Table II. The fit is shown together with the data in Fig. 2, for the case of the $250 \times 250$ lattice (with $m_{0}=0.02$ ).

We thus see that, unlike the magnetization $M$, the order parameter $\Omega$ is not scale-free at short times, but has a time scale, given by $\tau(L)$. The coefficients $a$ and $b$ seem to be independent of $L$ and related only to $m_{0}$. We have fitted the time scale

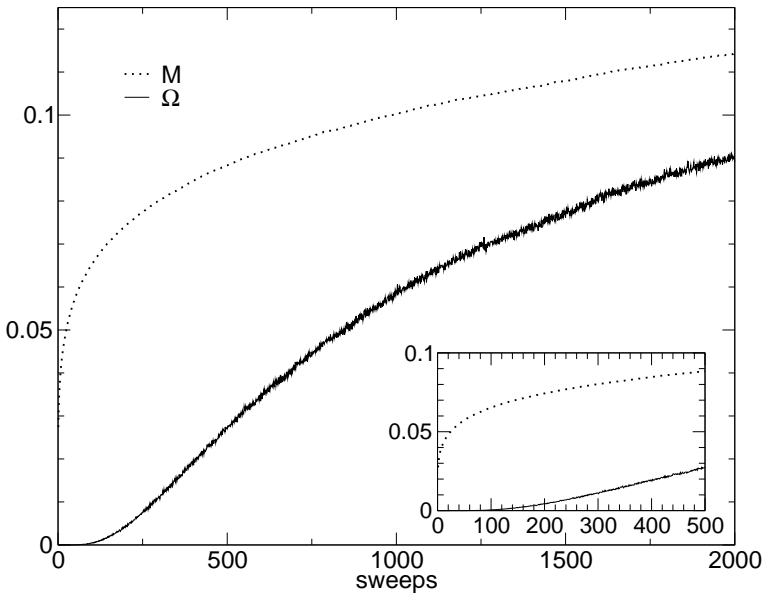

FIG. 1: Plot of the early time evolution of the magnetic $(M)$ and percolation $(\Omega)$ order parameters, for the case $L=250$ and $m_{0}=$ 0.02 .

TABLE I: The exponent $\theta$ as obtained from power-law fits for the magnetization $M$. The fit intervals are roughly $\left[t_{m i c}, 100\right]$, with $t_{m i c}$ between 5 and 10. The values of $\chi^{2} /$ d.o.f. are of order 1 .

\begin{tabular}{cccc}
$\mathrm{L}$ & $m_{0}=0.04$ & $m_{0}=0.03$ & $m_{0}=0.02$ \\
\hline 100 & $0.1820(5)$ & $0.1897(7)$ & $0.1850(9)$ \\
\hline 125 & $0.1831(4)$ & $0.1843(5)$ & $0.1859(7)$ \\
\hline 200 & $0.1836(3)$ & $0.1831(3)$ & $0.1842(7)$ \\
\hline 250 & $0.1863(6)$ & $0.186(1)$ & $0.186(2)$
\end{tabular}

$\tau$ in Eq.(6) to a power law with the lattice side $L$, obtaining good agreement and an exponent consistent with the dynamic critical exponent $z$ for the model, suggesting that the relevant time scale at short times is given by the relaxation time to equilibrium. A fit of $\tau$ to the form $L^{z}$ is shown in Fig. 3.

Finally, we have also looked at the observable given by

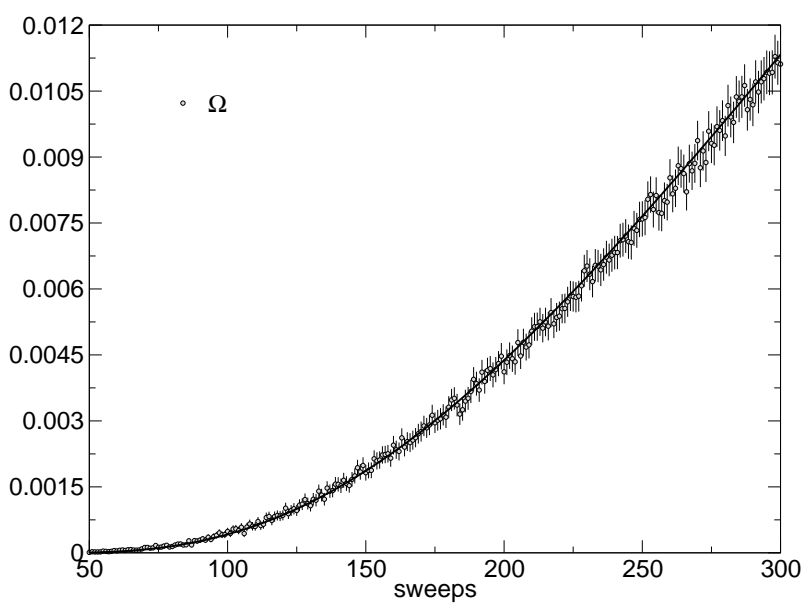

FIG. 2: Fit of the early time evolution of $\Omega$ to the form in Eq. (6), for the case $L=250$ and $m_{0}=0.02$. 


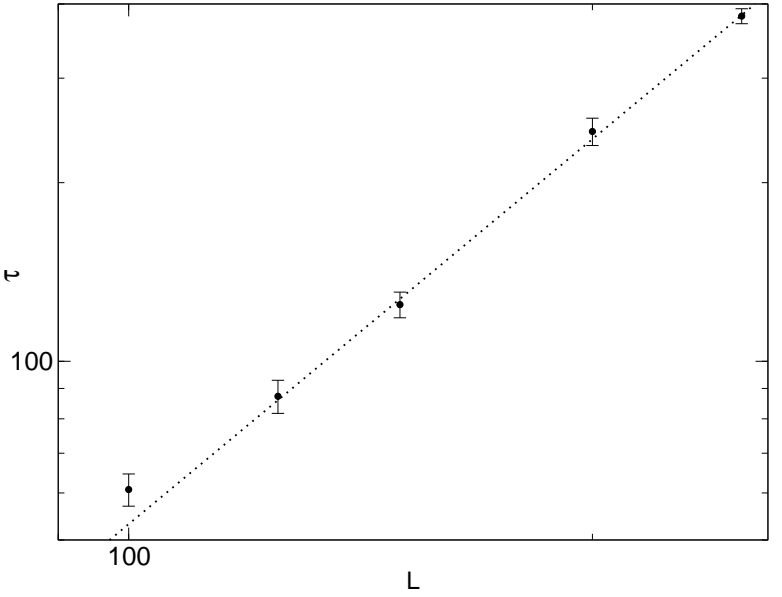

FIG. 3: Plot of $\tau(L)$ for $m_{0}=0.02$. The fit gives $\tau \sim L^{z}$ with $z=$ 2.15(4).

TABLE II: Results from fits of $\Omega$ to the form in Eq. (6). The fit intervals are roughly given by $\left[t_{m i c}, 90\right]$, with $t_{\text {mic }}$ between 5 and 10 .

\begin{tabular}{ccccc}
$\mathrm{L}$ & \multicolumn{4}{c}{$m_{0}=0.02$} \\
\hline & $\mathrm{a}$ & $\tau$ & $\mathrm{b}$ & $\chi^{2}$ \\
\hline 100 & $0.019(5)$ & $60(4)$ & $0.059(3)$ & 0.36 \\
\hline 125 & $0.013(3)$ & $87(6)$ & $0.061(1)$ & 0.37 \\
\hline 150 & $0.012(2)$ & $124(6)$ & $0.066(1)$ & 0.26 \\
\hline 200 & $0.017(4)$ & $243(12)$ & $0.07(1)$ & 0.43 \\
\hline 250 & $0.017(2)$ & $382(11)$ & $0.082(5)$ & 0.30
\end{tabular}

the pressure of the largest cluster of the lattice. This observable too is equivalent to the magnetization in the equilibrium regime, with the advantage that it is always well defined, whereas a spanning cluster may not occur or may occur more than once for a given (finite-volume) lattice configuration. (Nevertheless, we have checked that in our runs there is always at most one spanning cluster.) Similarly to $\Omega$, this observable does not show a power-law behavior, "lagging" behind the magnetization for early simulation times. The early dynamic behavior in this case is already reasonably well fitted by an exponential approach to the equilibrium value (analogous to the one found for cluster numbers in [9]), thus depending on a relaxation time scale.

\section{CONCLUSIONS}

We have investigated numerically the critical heat-bath dynamics for magnetic and percolation order parameters in the two-dimensional Ising model at short Monte Carlo times, starting from a small magnetization $m_{0}$. From our results we see that although the equilibrium behaviors of the magnetization $M$ and of the strength of the percolating cluster $\Omega$ are equivalent (see e.g. [4] and references therein), the two order parameters show qualitatively different dynamic critical behavior at short times. Whereas the magnetic order parameter $M$ shows a power-law behavior with the exponent $\theta$, one finds that $\Omega$ has a time scale, given by $\tau$ in Eq. (6), which seems to be related to the relaxation time of the algorithm. We are currently investigating the possibility that the observable $\Omega$ may suffer from finite-size effects related to the difficulty in forming a percolating cluster at the early stages of the simulation [10].

\section{Acknowledgments}

The authors thank Attilio Cucchieri for helpful comments and suggestions. This work was supported by FAPESP, CAPES and CNPq.
[1] D. Stauffer and A. Aharony, Introduction to Percolation Theory (Taylor \& Francis, 1992).

[2] H. Satz, Comp. Phys. Comm. 147, 46 (2002).

[3] P.W. Kasteleyn and C.M. Fortuin, J. Phys. Soc. Japan (Suppl.) 26, 11 (1969); A. Coniglio and W. Klein, J. Phys. A 13, 2775 (1980).

[4] W.G. Wanzeller, A. Cucchieri, G. Krein, and T. Mendes, Braz. J. Phys. 34, 247 (2004).

[5] P. Blanchard et al., J. Phys. A 33, 8603 (2000).

[6] W.G. Wanzeller, G. Krein, and T. Mendes, AIP Conf. Proc.
739, 608 (2005).

[7] H.K. Janssen, B. Schaub, and B. Schmittmann, Z. Phys. B 73, 539 (1989).

[8] J.-B. Zang et al., Phys. Lett. A 262, 226 (1999).

[9] D. Stauffer, Physica A 186, 197 (1992).

[10] W.G. Wanzeller, T. Mendes, and G. Krein. Short-time Dynamics of Percolation Observables, in preparation.

[11] Preliminary results were presented in [6]. 